\title{
Downregulation of G-protein-coupled receptor 30 in the hippocampus attenuates the neuroprotection of estrogen in the critical period hypothesis
}

\author{
YINGXI WU*, DAYUN FENG*, JIAJI LIN*, YAN QU, SHIMING HE, \\ YUAN WANG, GUODONG GAO and TIANZHI ZHAO
}

Department of Neurosurgery, Tangdu Hospital of The Fourth Military Medical University, Xi'an, Shaanxi 710038, P.R. China

Received May 26, 2017; Accepted January 3, 2018

DOI: $10.3892 / \mathrm{mmr} .2018 .8618$

\begin{abstract}
The aim of the present study was to investigate the role of G-protein-coupled receptor 30 (GPR30) in long-term $17 \beta$-estradiol (E2) deprivation (LTED) in a rat model with global cerebral ischemia (GCI), and its therapeutic target for ischemic stroke in the clinical setting. Following bilateral ovariectomy, GCI was induced in rats 1 or 10 weeks post-surgery. To determine the protein and mRNA expression levels of GPR30 in the hippocampal CA1 region of LTED rats, short-term E2 deprivation (STED) rats and naturally aging rats, western blot analysis and reverse transcription-quantitative polymerase chain reaction were performed. The results of the present study demonstrated that E2 treatment revealed significant neuroprotection post-GCI in STED rats, but not in LTED rats, as well as a decrease in the expression levels of GPR30 in the hippocampal CA1 region. In LTED rats,. Notably, no effects were observed on the ubiquitination of GPR30 following investigation in STED or LTED rats. While the protein and mRNA expression levels of GPR30 were also decreased in the hippocampal CA1 region of female 24-month-old rats compared with 3-month-old rats. E2 treatment initiated for the entire ovariectomy period elevated GPR30 mRNA and protein
\end{abstract}

Correspondence to: Dr Guodong Gao or Dr Tianzhi Zhao, Department of Neurosurgery, Tangdu Hospital of The Fourth Military Medical University, 1 Xin-si Road, Xi'an, Shaanxi 710038, P.R. China E-mail: gguodong@fmmu.edu.cn

E-mail: zhaotianzhi1981@163.com

*Contributed equally

Abbreviations: GPR30, G-protein-coupled receptor 30; E2, 17b-estradiol; LTED, long-term E2 deprivation; STED, short-term E2 deprivation; WHI, Women's Health Initiative; GCI, global cerebral ischemia; $\mathrm{ER} \alpha$, estrogen receptor- $\alpha$; $\mathrm{ER} \beta$, estrogen receptor- $\beta$; CCA, common carotid arteries; DMSO, dimethyl sulfoxide; IP, immunoprecipitation

Key words: estradiol, G-protein-coupled receptor 30, hippocampus, neuroprotection, G1 agonist, G15 antagonist expression levels, and attenuated the loss of hippocampal neurons in the GCI-induced CA1 region, indicating that E2 treatment exerted robust neuroprotection within LTED rats. However, the neuroprotective effect of E2 may be blocked by G15. The results of the present study revealed that downregulation of GPR30 expression may attenuate the neuroprotection of E2 within LTED conditions in rats post-ovariectomy by leading to neuronal insensitivity to E2 neuroprotection following cerebral ischemia. These results provide evidence that GPR30 may have potential as a novel therapeutic target for the treatment of clinical ischemic stroke.

\section{Introduction}

Previous studies have demonstrated that $17 \beta$-estradiol (E2) exhibits a neuroprotective effect in cerebral stroke and neurodegenerative diseases such as ischemic stroke and Alzheimer's disease (1-3). However, the Women's Health Initiative (WHI) trial did not reveal any decrease in stroke-associated morbidity in women that received hormone therapy $(4,5)$. An average age of 63.3 years for participants in the WHI is the most important criticism for WHI, which far exceeds the menopause age (6). The 'critical period hypothesis' has been proposed, which describes a precise window of opportunity for beneficial hormone therapy following menopause $(7,8)$. Estrogen may be administered prior to or during perimenopause to protect the neural and cardiovascular systems $(7,9)$. Previous studies in animals also provided supporting information for the period hypothesis and evidence to support neurological consequences of long-term E2 deprivation (LTED; 10 weeks) (10-13). G-protein-coupled receptor 30 (GPR30), also termed G-protein-coupled estrogen receptor 1 , is a recently identified G-protein-coupled receptor that closely binds estrogen and is characterized as an estrogen receptor within the membrane. The GPR30 results in rapid non-genomic signaling events and transcriptional regulation (14). Previous studies have demonstrated the neuroprotective effects of GPR30 using the GPR30-selective agonist, G1 $(15,16)$. In vivo studies in female mice using global cerebral ischemia (GCI) and middle cerebral artery occlusion models also demonstrated that G1 exhibited neuroprotective effects against cerebral ischemia $(17,18)$. Furthermore, a 
previous study that employed an in vivo antisense oligodeoxynucleotide knockdown approach revealed the important role of GPR30 in mediating rapid kinase activation and neuroprotection by E2 in an animal model of GCI (19). In addition, the rapid signaling effects of $\mathrm{G} 1$ in the activation of the prosurvival kinases Akt and extracellular signal-regulated kinases (ERKs), and in the inhibition of the proapoptotic signaling kinase, c-Jun $\mathrm{N}$-terminal kinase, in the CA1 region of the hippocampus post-GCI were demonstrated (19). C-terminus of heat-shock cognate protein 70-interacting protein-mediated estrogen receptor (ER)- $\alpha$ degradation was reported within the CA1 region of the hippocampus following aging and LTED, which was associated with loss of estrogen-mediated neuroprotection (20).

However, alterations in the GPR30 expression levels and the effect of GPR30 on the loss of estrogen-mediated neuroprotection in LTED and naturally aging female rats remain largely unknown. Therefore, the present study aimed to investigate the role of GPR30 in LTED in a GCI rat model and its potential in the treatment of clinical ischemic stroke.

\section{Materials and methods}

Animal preparation. A total of 322 adult female Sprague-Dawley rats (12-weeks old, 250-300 g) were purchased from the Experimental Animal Center of the Fourth Military Medical University (Xi'an, China). The study protocol was approved by the Animal Care and Use Committee of the Fourth Military Medical University and animal use conformed to the National Institutes of Health Guidelines for the Care and protection of Laboratory Animals (21). Rats were randomly divided into sham $(n=21)$, STED $(n=49)$, LTED $(\mathrm{n}=181), 3$ month-old $(\mathrm{n}=22$, female Sprague-Dawley rats, 250-300 g) and 24 month-old ( $n=49$, female Sprague-Dawley rats, 450-500 g; both 3 and 24 month-old rats were purchased from the Experimental Animal Center of the Fourth Military Medical University) groups. Rats were allowed free access to water and food until optimal operation conditions and were maintained under a $12 \mathrm{~h}$ light/dark cycle at room temperature (RT) and 50\% humidity.

Ovariectomy and GCI. Following bilateral ovariectomy, GCI was induced with four-vessel occlusion in rats at 1 week (for STED group) or 10 weeks (for LTED group) post-surgery, as previously described (22-24). Briefly, the rats were anesthetized with $10 \%$ chloral hydrate $(350 \mathrm{mg} / \mathrm{kg}$, intraperitoneal injection; no signs of peritonitis were observed) and the vertebral arteries were electrocauterized with a monopolar coagulator and the common carotid arteries (CCA) were exposed. After $24 \mathrm{~h}, \mathrm{CCA}$ were re-exposed and clipped for $10 \mathrm{~min}$ and rats were subsequently reperfused. The rats with loss of righting reflex, loss of response to light and dilated pupils within $30 \mathrm{sec}$ during ischemia were selected for subsequent experiments. During ischemia, a temperature of $37 \pm 0.5^{\circ} \mathrm{C}$ was maintained using a thermal blanket. The same surgical procedures were performed for animals in sham group, however, the CCA was not occluded.

Drug administration. In certain rats, an E2 Alzet minipump was implanted under the skin in the upper region of the mid-back under anesthesia. The LTED rats were received E2 treatment ( $0.025 \mathrm{mg} /$ day) for 10 weeks immediately post-ovariectomy, or for 1 week after 10 weeks post-ovariectomy, while the STED rats were administrated with once intracerebroventricular treatment of $\mathrm{E} 2(10 \mu \mathrm{M}$ in $5 \mu \mathrm{l}$ normal saline) $1 \mathrm{~h}$ pre-GCI induction. In certain STED or LTED rats, G1 (B5455; ApexBio, Houston, TX, USA; $50 \mu \mathrm{g}$ in $5 \mu \mathrm{l}$ dimethyl sulfoxide; DMSO), G15 (B5469; ApexBio; $100 \mu \mathrm{g}$ in $5 \mu \mathrm{l}$ DMSO) or E2-bovine serum albumin (E2-BSA; RPU50787; Biomatik, Wilmington, DE, USA; $10 \mu \mathrm{M}$ in $5 \mu$ l normal saline) was bilaterally administered using an intracerebroventricular injection $1 \mathrm{~h}$ pre-GCI (19). The drug procedures were same as above for 3-month and 24-month rats. In total, the injections were performed at $1 \mu \mathrm{l} / \mathrm{min}$ rate and the needle was withdrawn after $5 \mathrm{~min}$. During the whole operation, a temperature of $37 \pm 0.5^{\circ} \mathrm{C}$ was maintained. At the indicated time points, rats were sacrificed by decapitation under deep anesthesia and their brains were removed for the following experiments.

Western blot analysis and co-immunoprecipitation (co-IP). Protein samples were extracted after rats were sacrificed as previously described $(23,24)$. Briefly, tissues of the hippocampal CA1 region were microdissected on ice and homogenized in lysis buffer $(1: 10 \mathrm{w} / \mathrm{v})$ containing $50 \mathrm{mM} \mathrm{3-morpho-}$ linopropanesulfonic acid ( $\mathrm{pH} 7.4$ ), $150 \mathrm{mM} \mathrm{NaCl}, 20 \mathrm{mM}$ $\beta$-glycerophosphate, $3 \mathrm{mM}$ DL-dithiothreitol, $2 \mathrm{mM} \mathrm{Na} \mathrm{VO}_{4}$, $1 \mathrm{mM}$ EGTA, $1 \mathrm{mM}$ EDTA, $1 \mathrm{mM} \mathrm{NaF}, 1 \%$ Triton-X-100, $1 \%$ NP-40 and protease inhibitor $(0.5 \mathrm{mM}$ phenylmethanesulfonyl fluoride, $10 \mathrm{mg} / \mathrm{ml}$ each of aprotinin, leupeptin, and pepstatin A) for $10 \mathrm{~min}$. Following centrifugation at $15,000 \mathrm{xg}$ for $15 \mathrm{~min}$ at $4^{\circ} \mathrm{C}$, the supernatant was collected and protein was quantified using a bicinchoninic acid protein assay kit (Thermo Fisher Scientific, Inc., Waltham, MA, USA). Total $20 \mu \mathrm{g}$ proteins ere subsequently electrophoresed using 6-15\% SDS-PAGE and electro-transferred to polyvinylidene fluoride membranes. After blocking in 5\% nonfat milk for $1 \mathrm{~h}$ at RT, the membranes were incubated overnight at $4^{\circ} \mathrm{C}$ with the following primary antibodies: Anti-ubiquitin (1:1,000; cat. no. ab19247; Abcam, Cambridge, UK), anti-GPR30 (1:500; ab39742; Abcam) anti-ER $\alpha$ (1:500; cat. no. bs-0725R; BIOSS, Beijing, China), anti-ER $\beta$ (1:500; cat. no. bs-0255R; BIOSS) and anti-and GAPDH (1:5,000; cat. no. BM1623; Wuhan Boster Biological Technology, Ltd., Wuhan, China). The anti-mouse/rabbit HRP-conjugated IgG secondary antibodies (1:5,000; cat. nos. ab6728/ab6721; Abcam) were incubated for $1 \mathrm{~h}$ at RT. The blots were then detected using a Clarity ${ }^{\mathrm{TM}}$ western ECL kit (cat. no. 170-5060; Bio-Rad Laboratories, Inc., Hercules, CA, USA) with an Odyssey Imaging system (LI-COR Biosciences, Lincoln NE, USA) was used for the detection of blots and ImageJ software (version 1.47; National Institutes of Health, Bethesda, MD, USA) was used to quantify protein expression.

For co-IP detection, the supernatant fractions were diluted 4-fold with HEPES buffer and were incubated with anti-GPR30 (1:100; cat. no. ab39742; Abcam) and normal IgG antibody (5 $\mu \mathrm{g}$, Cell Signaling Technology, Inc.) for $4 \mathrm{~h}$ at $4^{\circ} \mathrm{C}$. Samples were pre-incubated with $20 \mu \mathrm{l}$ protein $\mathrm{A} / \mathrm{G}$ agarose (Santa Cruz Biotechnology, Inc., Dallas, TX, USA) for $60 \mathrm{~min}$ at $4^{\circ} \mathrm{C}$, and then centrifuged at $1,000 \mathrm{x} \mathrm{g}$ for $30 \mathrm{~min}$ at $4^{\circ} \mathrm{C}$ to remove nonspecifically-adhered proteins. 
Western blotting was used to analyze the precipitated protein.

Immunofluorescence. Histological assessment was performed in fixed for $2 \mathrm{~h}$ (4\% paraformaldehyde in 0.01 M PBS, RT) and frozen hippocampal brain sections $(25,26)$. Briefly, brain sections $(25 \mu \mathrm{m})$ were permeabilized with $0.4 \%$ Triton-X-100 in PBS for $30 \mathrm{~min}$ and blocked with $5 \%$ normal serum for $30 \mathrm{~min}$ at RT. Then the sections were incubated at $4^{\circ} \mathrm{C}$ overnight with diluted GPR30 antibody (1:50; cat. no. ab39742; Abcam). Following three washes with PBS, the sections were incubated for $2 \mathrm{~h}$ at RT with goat anti-rabbit immunoglobulin G-Alexa Fluor 488 secondary antibody (1:300; cat. no. A-11008; Invitrogen; Thermo Fisher Scientific, Inc.). Fluorescent images were captured using a confocal laser microscope system at 488 nm wavelength (FV1000; Olympus Corporation, Tokyo, Japan).

Nissl staining and neuron survival quantification. At 7 days post-GCI, $0.9 \%$ saline and then $4 \%$ paraformaldehyde (ice-cold) in $0.1 \mathrm{M}$ phosphate buffer were used for perfusion. Brains were removed and placed in formalin at $4^{\circ} \mathrm{C}$ overnight, fixed in $30 \%$ sucrose in phosphate buffer at $4^{\circ} \mathrm{C}$ for up to two days and longitudinally cut into $25-\mu \mathrm{m}$ sections. Coronal sections were stained with $0.1 \%$ cresyl violet for $2 \mathrm{~min}$ at RT, dehydrated using a graded alcohol series $(100$, 90,80 , and $70 \%$ ), placed in xylene and covered with a coverslip following the addition of HistoMount medium (Thermo Fisher Scientific, Inc.). And slices were then mounted with Lab Vision ${ }^{\text {TM }}$ Ultramount resin (TA-060-UM; Thermo Fisher Scientific, Inc.). The sections were evaluated using a light microscope (DFC310FX; Leica Microsystems, Inc., Buffalo Grove, IL, USA). Viable CA1 neurons within each rat were counted bilaterally in at least five sections $(250 \times 250 \mu \mathrm{m}$ per view). Viable neurons exhibited rounded cell shapes and clearly visible nucleoli. Pyknotic and shrunken neurons were not counted. Cell counts were averaged from bilateral hippocampi on five sections. Data from each group were presented as the mean \pm standard deviation.

Reverse transcription-quantitative polymerase chain reaction (RT-qPCR). The STED (1 week post-surgery), LTED (10 week post-surgery), 3-month and 24-month rats were sacrificed, and hippocampalCA1 region tissues were collected and total RNA was isolated using the RNAqueous-4PCR kit (Ambion; Thermo Fisher Scientific, Inc.), according to the manufacturer's protocol. First strand cDNA was reverse transcribed from $500 \mathrm{ng}$ total RNA with a High Capacity cDNA Archive kit at $37^{\circ} \mathrm{C}$ for $15 \mathrm{~min}$ and $85^{\circ} \mathrm{C}$ for $5 \mathrm{sec}$ (Applied Biosystems; Thermo Fisher Scientific, Inc.). qPCR reactions were performed with $50 \mathrm{ng}$ cDNA on an ABI Prism 7000 sequence detection system (Applied Biosystems; Thermo Fisher Scientific, Inc.) in triplicate using a SYBR Premix Ex Taq kit (cat. no. 11780200; Invitrogen; Thermo Fisher Scientific, Inc). Primers used were synthesized by Invitrogen (Thermo Fisher Scientific, Inc.) as follows: GPR30, 5'-GCA GAAAGCCCTGAGGATGAT-3' (forward) and 5'-ATGGCA GAAGCGGTTGAGAG-3' (reverse); and GAPDH, 5'-TGT GTCCGTCGTGGATCTGA-3' (forward) and 5'-TTGCTG TTGAAGTCGCAGGAG-3' (reverse). Amplification and detection were performed under the following conditions: Incubation at $95^{\circ} \mathrm{C}$ for $30 \mathrm{sec}$, followed by 40 cycles of denaturation at $95^{\circ} \mathrm{C}$ for $5 \mathrm{sec}$ and subsequent annealing and extension at $60^{\circ} \mathrm{C}$ for $30 \mathrm{sec}$. For each sample, GAPDH expression was analyzed to normalize target gene expression. Relative gene expression was calculated using the $2^{-\Delta \Delta \mathrm{Cq}}$ method (27). Each sample was analyzed in triplicate.

Terminal deoxynucleotidyl-transferase-mediated dUTP nick end labelling (TUNEL) assay. The rat brains were harvested at 3 days post-GCI, and coronal cryosections $(25-\mu \mathrm{m})$ were obtained from $\geq 5$ rats per group. The number of apoptotic cells was determined via a TUNEL assay (cat. no. 11684795910; Roche Diagnostics, Basel, Switzerland) following the manufacturer's protocol. Five random fields in the penumbral areas of each section were observed with a confocal microscope (FV1000; Olympus Corporation, Tokyo, Japan) and TUNEL-positive cells in the cerebral ischemic penumbra were counted manually.

Serum estradiol concentration assay. For the sham, STED (1 week) and LTED (10 weeks) group, the tail blood samples of rats were collected on the first day pre-surgery and on the first, third, fifth and seventh day post-surgery. The rats were anesthetized by intraperitoneal injection $(0.35 \mathrm{ml} / 100 \mathrm{~g})$ with $10 \%$ chloral hydrate. The rats were fixed in the prone position and the tails were soaked in water at $50^{\circ} \mathrm{C}$ for several minutes until the tail vessels were filling. After alcohol disinfection, the tail was cut with a surgical scissors for 5-10 $\mathrm{mm}$ and the blood flowed into the EP tube. Blood specimens of $1 \mathrm{ml}$ were collected each time. After the blood samples were labeled, they were allowed to stand overnight at $4^{\circ} \mathrm{C}$ and centrifuged at $3,000 \mathrm{x} \mathrm{g}$ for $10 \mathrm{~min}$. The supernatant was collected in an EP tube and stored at $-80^{\circ} \mathrm{C}$ freezer. The collected specimens were sent to the laboratory, using Roche E601 electrochemical luminescence automatic analyzer to detect concentration of serum estradiol.

Statistical analysis. All data are presented as the mean \pm standard deviation. One-way or two-way analysis of variance was performed followed by the Bonferroni/Dunn post-hoc tests to determine group differences using GraphPad Prism software (GraphPad Software 6.0; GraphPad Software, Inc., Inc., La Jolla, CA, USA). When only two groups were compared, a Student's t-test was performed for comparison between two groups. $\mathrm{P}<0.05$ was considered to indicate a statistically significant difference.

\section{Results}

No neuroprotection by E2 or GI in the CA1 region of the hippocampus in LTED rats. The effects of E2 or G1 on E2-associated neuroprotection in the STED and LTED rats following GCI were analyzed. Hippocampal sections were obtained from rats at 7 days reperfusion post-GCI and Nissl's staining was conducted to detect surviving neurons. GCI (Pla) led to a marked decrease in the number of surviving neurons within the CA1 region at 7-days-reperfusion in the Pla group, compared with in the sham group (Fig. 1). Neuroprotective effects on the hippocampal CA1 region in STED rats were 
A

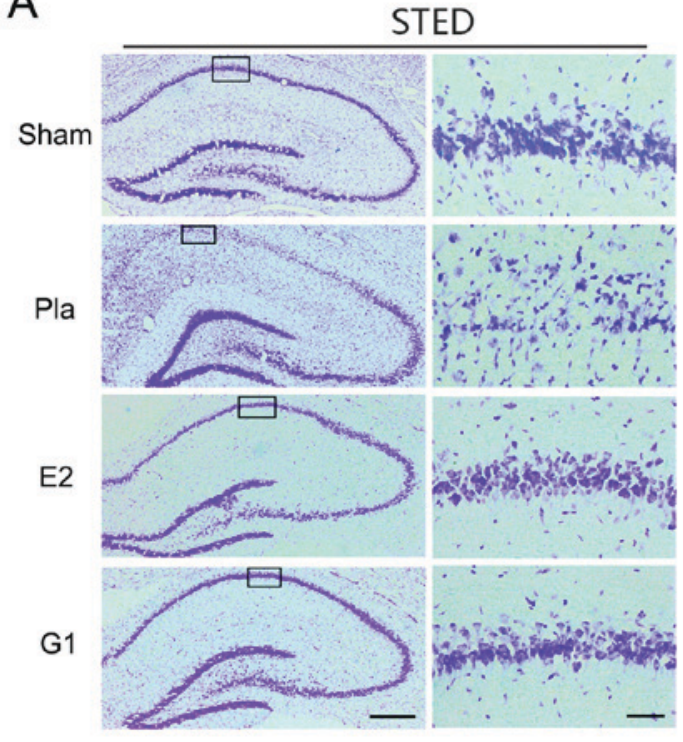

B

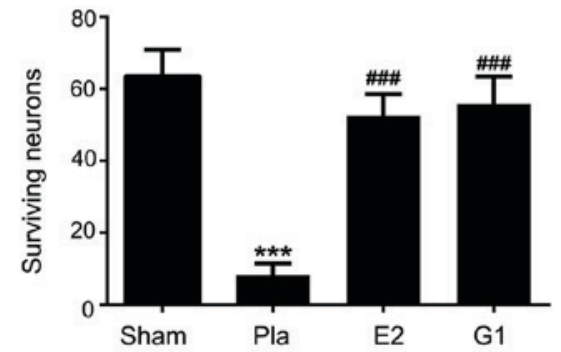

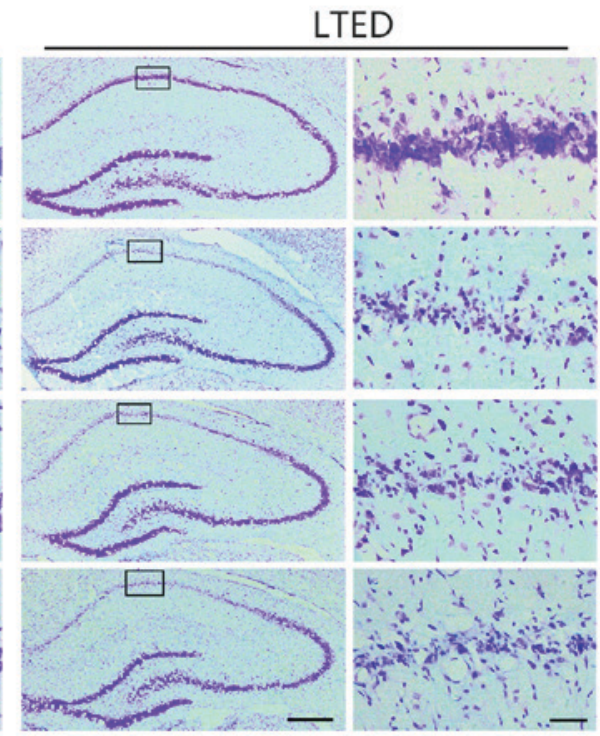

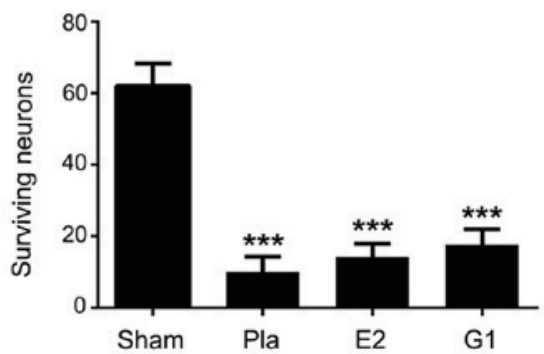

Figure 1. Neuroprotection of G1 and E2 in the hippocampal CA1 region within STED but not LTED rats following global cerebral ischemia. (A) Nissl's staining photomicrographs of the hippocampus in sham, Pla-, E2- and G1-treated ovariectomized rats of STED and LTED following ischemic reperfusion for 7 days. The surviving CA1 pyramidal cells demonstrated intact and round nuclei following ischemia and were counted as surviving cells. Scale bar $=500 \mu \mathrm{m}$ (left column) and $50 \mu \mathrm{m}$ (right column) in STED and LTED group. (B) Data revealed the number of surviving neurons per $250 \mu \mathrm{m}$ length of medial CA1. Data are presented as the mean \pm standard deviation, $\mathrm{n}=8$ rats per group. ${ }^{* * *} \mathrm{P}<0.001$ vs. sham group; ${ }^{\# \# \#} \mathrm{P}<0.001$ vs. Pla group. G1, G-protein-coupled receptor 30-selective agonist; E2, 17b-estradiol; Pla, placebo; NeuN, neuronal nuclei.
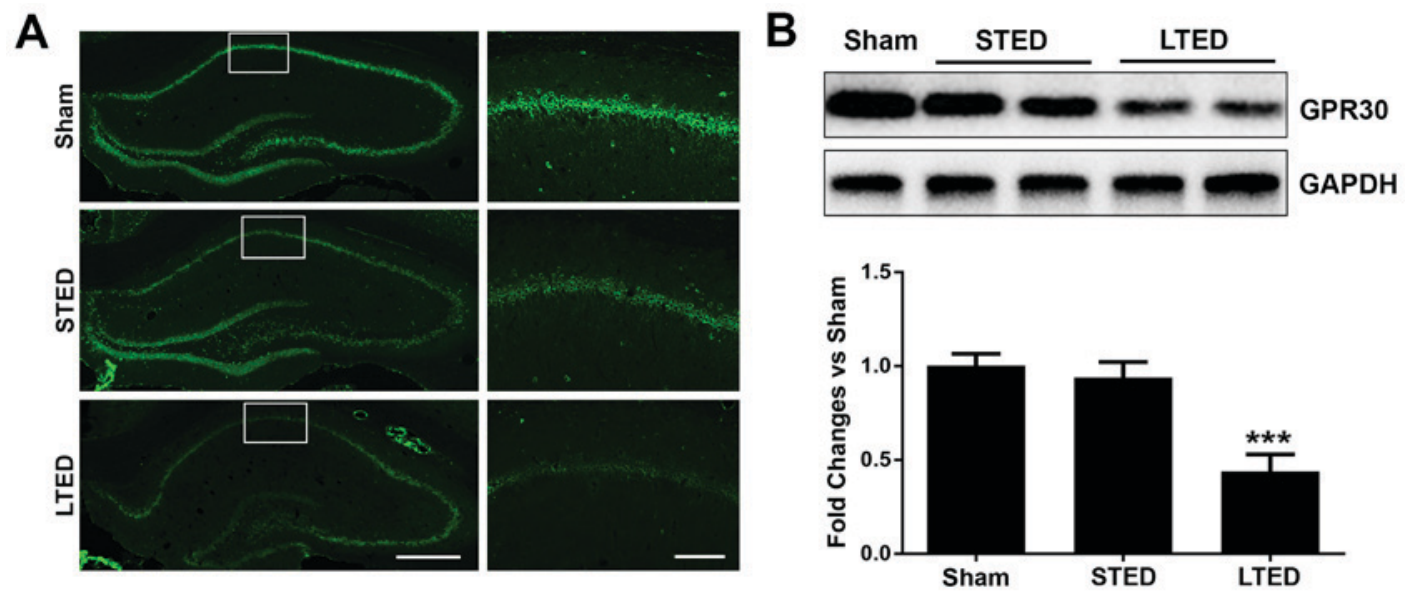

Figure 2. Immunofluorescence of GRP30 and GPR30 protein expression levels within the hippocampal CA1 region of STED and LTED rats. (A) Immunofluorescence staining presented GPR30 expression in the hippocampal CA1-3 and dentate gyrus regions of the sham group. GPR30 immunoreactivity in the LTED rats was markedly lower compared with sham and STED rats. Scale bar=500 $\mu \mathrm{m}$ (left column) and $100 \mu \mathrm{m}$ (right column). (B) Western blot analysis of the expression of total GPR30 and GAPDH was performed in sham, STED (7 days post-) and LTED (10-week later) groups. Data are presented as the mean \pm standard deviation, $\mathrm{n}=8$ rats per group. ${ }^{* * *} \mathrm{P}<0.001$ vs. STED group. GPR30, G-protein-coupled receptor 30; E2, 17b-estradiol; STED, short-term E2-deprivation; LTED, long-term E2 deprivation.

observed in the G1 and E2 treatment groups, as indicated by Nissl's staining and quantification of surviving neurons (Fig.1).
However, E2 or G1 treatment post-GCI revealed no neuroprotective effects on the hippocampal CA1 region in LTED 
rats (Fig. 1). These results indicated $\mathrm{E} 2$ or $\mathrm{G} 1$ exerted robust neuroprotection in STED rats, but exerted no neuroprotection in LTED rats.

GPR30 expression is reduced in the CAl region of the hippocampus in LTED rats. Immunofluorescence and western blot analysis were performed to investigate the protein expression levels of GPR30 in the CA1 region of the hippocampus in STED and LTED rats. Immunofluorescence detected high levels of GPR30 immunoreactivity in the CA1 region of the hippocampus in the sham group; the results demonstrated a marked difference between LTED and STED rats, with reduced levels in the LTED rats (Fig. 2A). Furthermore, western blotting analysis revealed that GPR30 expression levels in the CA1 region of the hippocampus were significantly reduced in LTED rats compared with STED rats (Fig. 2B). The mean serum levels of E2 in the sham, STED (1 week) and LTED (10 weeks) groups are presented in Table I. Levels of E2 within rats that received ovariectomies (STED and LTED) were markedly lower compared with the sham group (Table I). In short, these results indicated GPR30 decreased in the CA1 region of the hippocampus in LTED rats compared with STED rats.

$m R N A$ levels and ubiquitination of GPR30 in the CA1 region of the hippocampus in LTED rats. mRNA and ubiquitination levels of GPR30 were determined in STED and LTED rats. The RT-qPCR results demonstrated that GPR30 mRNA expression levels within STED rats were $>2$ times higher compared with those in LTED rats (Fig. 3A). Further analysis revealed that the ubiquitination of GPR30 exhibited no significant difference in LTED rats compared with STED rats (Fig. 3B). Reverse IP with ubiquitin and blotting for GPR30 indicated marginal alterations in GPR30 ubiquitination within LTED rats compared with STED rats (Fig. 3C). The decrease of Ub GPR30 in Fig. 3C was due to the downregulation of GPR30 expression in hippocampus CA1. There was no significant change of GPR30 ubiquitination among the sham, STED and LTED groups. The decrease of GPR30 was attributable to the decreased mRNA levels but not the ubiquitination of GPR30.

Effects of E2 treatment 10-weeks post-ovariectomy or during the ovariectomy period on GPR30 expression levels and hippocampal neuronal survival post-GCI in LTED rats. E2-associated effects on the protein and mRNA expression levels of GPR30 were tested about E2 treatment 10 weeks post-ovariectomy and for the entire ovariectomy period (10 week). E2 treatment initiated at 10 weeks post-ovariectomy revealed no effects in preventing reductions in GPR30 protein expression levels within the CA1 region of the hippocampus (Fig. 4A). In rats that received E2 treatment for the whole ovariectomy period (10 week), E2 prevented a decrease in the GPR30 protein and mRNA expression levels in the CA1 region of LTED rats (Fig. 4B and C). TUNEL analysis was performed at 3 days post-GCI to investigate apoptosis in the hippocampal CA1 region, and the results demonstrated that while the number of apoptotic cells was were only slightly decreased by E2 treatment that was initiated at 10 weeks post-ovariectomy compared with the Pla (GCI) group in LTED rats (Fig. 5A and B), the reduction observed following
Table I. Serum E2 levels in ovariectomized rats according to treatments.

A, Serum E2 levels in STED ovariectomized rats

\begin{tabular}{lcc}
\hline Treatment & Serum E2,pg/ml & P-value \\
\hline Sham & $25.2 \pm 1.8$ & $<0.001$ \\
STED & $5.3 \pm 0.6$ & \\
\hline
\end{tabular}

$\mathrm{B}$, Serum E2 levels in LTED ovariectomized rats

\begin{tabular}{lcc}
\hline Treatment & Serum E2,pg/ml & P-value \\
\hline Sham & $26.1 \pm 1.6$ & $<0.001$ \\
LTED & $0.8 \pm 0.2$ &
\end{tabular}

E2, 17b-estradiol; LTED, long-term E2 deprivation; STED, short-term E2-deprivation.

treatment with E2 immediately post-ovariectomy and maintained for 10 weeks was greater in LTED rats (Fig. 5C and D). Nissl's staining indicated that E2 treatment initiated ten weeks after ovariectomy did not exhibit robust neuroprotection within LTED rats following GCI (Fig. 5E). However, in rats treated with E2 immediately after ovariectomy and maintained for 10 weeks, E2 treatment exhibited marked neuroprotective effects in LTED rats, and the administration of G15 at the end of E2 treatment attenuated the neuroprotective effects of E2, as indicated by Nissl's staining (Fig. 5F). Quantification of surviving neurons confirmed these observations for both E2 treatment time-points (Fig. 5G and H). In conclusion, E2 treatment initiated immediately post-ovariectomy and maintained for 10 weeks can prevent the decrease of GPR30 and mRNA levels and can exhibit a marked neuroprotective effects in LTED rats.

Alterations of protein, $m R N A$ and ubiquitination levels of GPR30 and the survival of hippocampal neurons in naturally aging female rats. Subsequently, the protein expression levels of GPR30, ER $\alpha$ and ER $\beta$ were investigated in the CA1 region of the hippocampus between 3- and 24-month-old rats. Western blot analysis of GPR30 revealed that the protein expression levels of GPR30, ER $\alpha$ and ER $\beta$ were decreased significantly within 24-month-old rats compared with 3-month-old rats (Fig. 6A). The RT-qPCR results revealed that the mRNA expression levels of GPR30 within the CA1 region of the hippocampus of 24-month-old rats were decreased to $\sim 50 \%$ of the levels in 3-month-old female rats (Fig. 6B); however, the ubiquitination of GPR30 revealed no significant differences between 3- and 24-month-old rats (Fig. 6C). Furthermore, Nissl's staining results of the CA1 region of the hippocampus and quantification of neuronal survival demonstrated that G1 or E2 did not exhibit neuroprotection within the 24-month-old female rats (Fig. 6D and E). The decline of GPR30 resulted in the loss of E2 neuroprotection in aging female rats, and these results further confirmed the mechanism of the decrease of GPR30. 
B

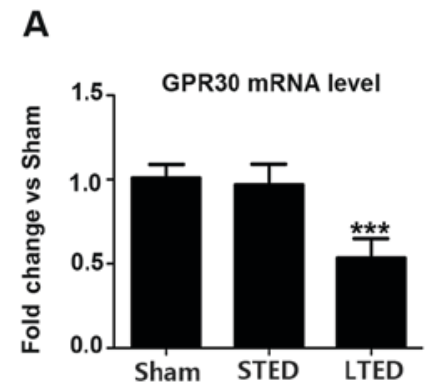

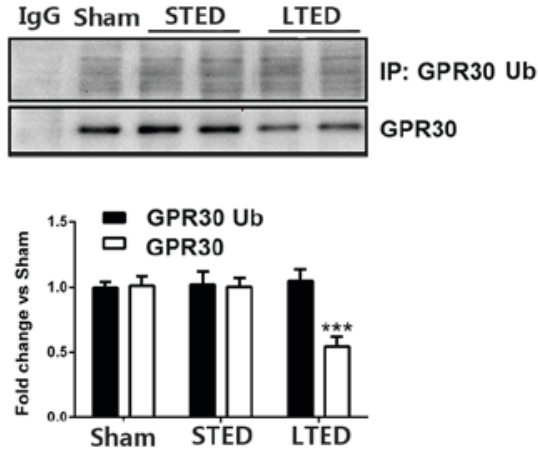

C

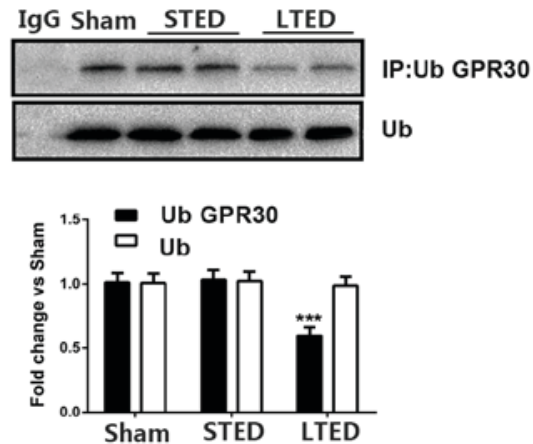

Figure 3. LTED significantly decreased GPR 30 mRNA expression levels. (A) Quantitative analysis of the mRNA expression of GPR30 was performed using reverse transcription-quantitative polymerase chain reaction of the hippocampal CA1 region in sham, STED, and LTED rats. A significant reduction was observed in GPR30 mRNA expression levels in the LTED group compared with the STED group. (B) IP using anti-GPR30 antibody or nonspecific IgG was conducted for hippocampal CA1 protein samples from sham, STED, and LTED rats at $3 \mathrm{~h}$ after reperfusion, and blotting using anti-ubiquitin or anti-GPR 30 antibodies was performed. No alterations were observed in the ubiquitination of GPR30 in LTED rats compared with STED rats at $3 \mathrm{~h}$ following reperfusion. (C) In reverse co-IP experiments, samples were subjected to IP with anti-ubiquitin antibody; immunocomplexes were probed for the presence of GPR30 and ubiquitination as indicated. Data are presented as the mean \pm standard deviation, $n=7-8$ rats per group, and fold change vs. the control group. ${ }^{* * * *} \mathrm{P}<0.001$ vs. STED group. E2, 17b-estradiol; LTED, long-term E2 deprivation GPR30, G-protein-coupled receptor 30; STED, short-term E2-deprivation; IP, immunoprecipitation; IgG, immunoglobulin G; Ub, ubiquitin.

A
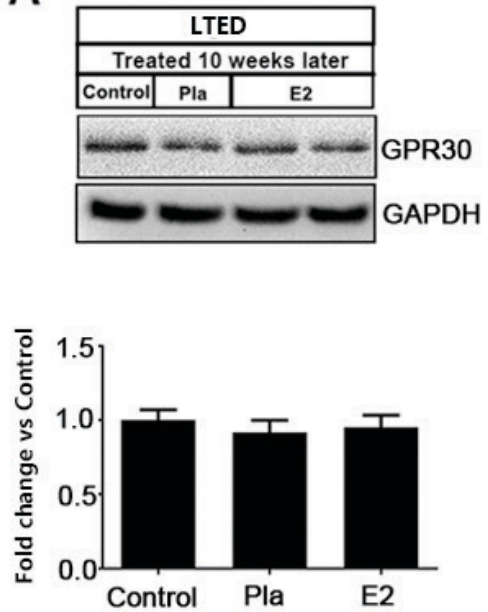

B
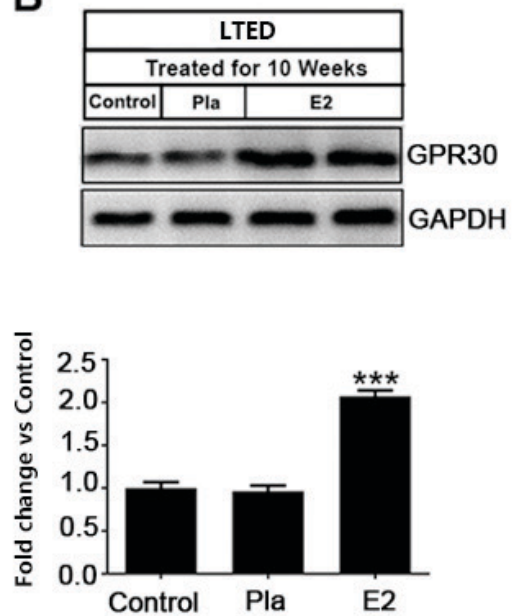

C

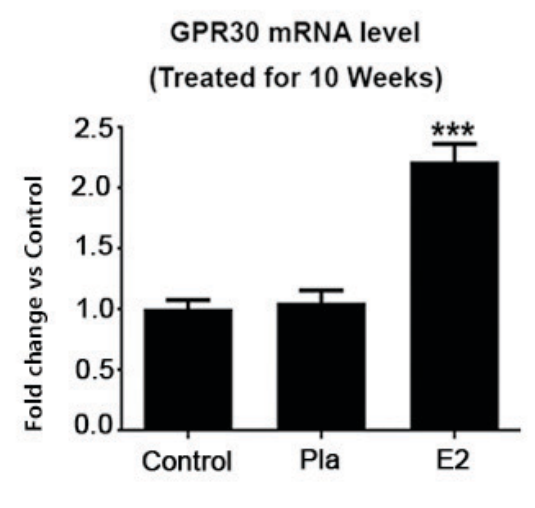

Figure 4. E2 treatment for the entire ovariectomy period prevents decreases in the protein and mRNA expression levels of GPR30. Western blotting for total GPR30 and GAPDH was performed on hippocampal CA1 protein following Pla or E2 treatment at (A) 10 weeks post-ovariectomy and (B) immediately after ovariectomy and maintained for 10 weeks. The decrease in GPR30 was inhibited in rats that were treated immediately after ovariectomy for 10 weeks but not in rats treated at 10 weeks post-ovariectomy. (C) Reverse transcription-quantitative polymerase chain reaction was conducted for the quantitative analysis of the mRNA expression levels of GPR30 within sham, Pla and E2 group rats. E2 replacement for 10 weeks immediately post-ovariectomy inhibited the decrease in GPR30 mRNA expression levels. Data are presented as the mean \pm standard deviation, $n=6-8$ per group. ${ }^{* * *} \mathrm{P}<0.001$ vs. the pla group. E2, $17 b$-estradiol; GPR30, G-protein-coupled receptor 30; Pla, Placebo.

\section{Discussion}

Estrogen and its receptor GPR30 have been revealed to exert neuroprotective effects against cerebral stroke and neurodegenerative diseases. However, their roles and mechanisms following ischemic stroke during the critical period remain unknown. The present study confirmed that E2 and G1 exerted marked neuroprotective effects against cerebral ischemia in STED rats following GCI, consistent with previous reports $(18,28)$; however, similar effects were not observed within LTED rats. Additionally, GPR30 expression levels were decreased within LTED rats, which may be primarily attributable to the decline of GPR30 mRNA expression levels, and not the degradation of GPR30 via ubiquitination. Importantly, E2 treatment during the entire ovariectomy period for 10 weeks rather than after 10-week ovariectomy exerted robust neuroprotection in LTED rats. Therefore, we hypothesized that the reduction in GPR30 expression may attenuate the neuroprotective effects of E2, which may occur via neuronal insensitivity to E2 following cerebral ischemia during the critical period. In addition, GPR30 may have potential as an effective therapeutic target against clinical ischemic stroke during the critical period.

The present study provided important evidence for the hypothesis of the beneficial role of the E2 replacement in critical period which states that a precise window of opportunity 
A

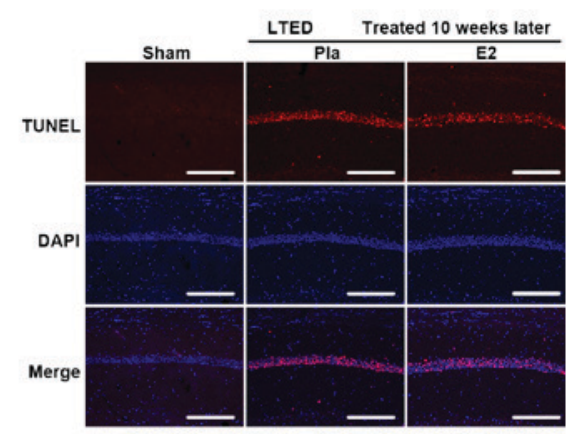

C

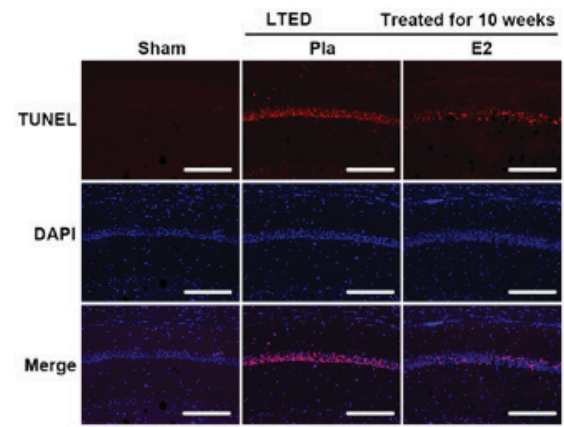

B

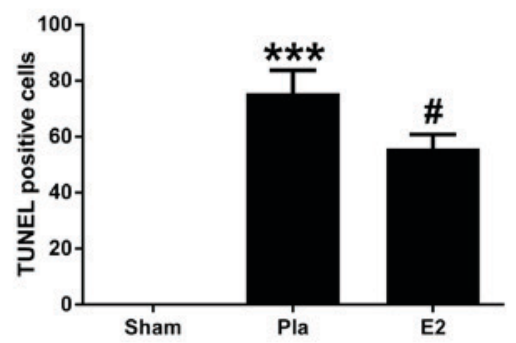

D

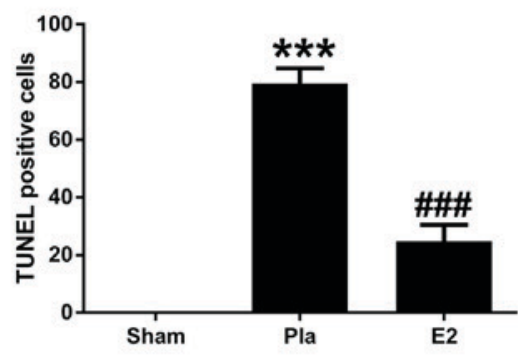

Treated 10 weeks later

E

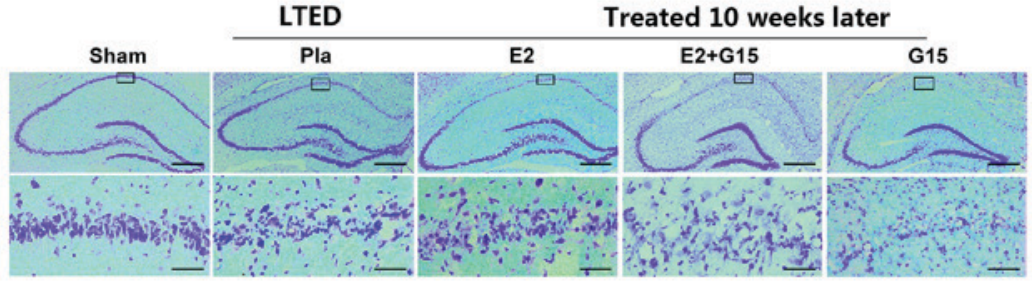

$\mathbf{F}$

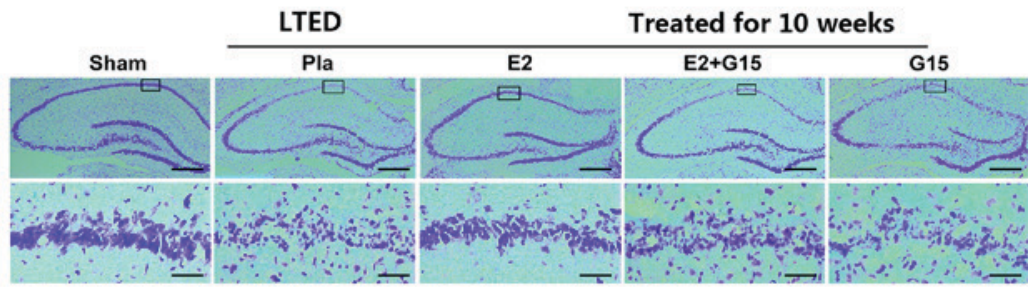

G

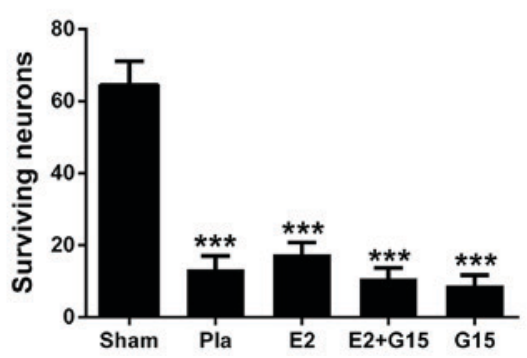

H

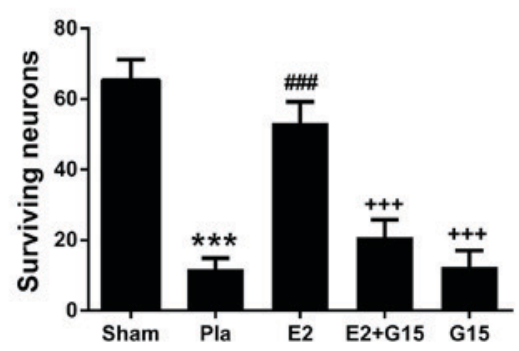

Figure 5. Effects of E2 treatment initiated at 10 weeks post-ovariectomy or immediately after ovariectomy on hippocampal neuron survival and apoptosis post-global cerebral ischemia. TUNEL staining was performed at 3 days following reperfusion. (A) Typical photomicrographs of the hippocampal CA1 region from rats that received E2 treatment at 10 weeks post-ovariectomy. TUNEL-positive cells stained red and all other cells were stained blue with DAPI. Scale bar $=100 \mu \mathrm{m}$. (B) TUNEL-positive cells were quantified in each group, $\mathrm{n}=8$ rats per group. Data is presented as the average number of TUNEL-positive cells in five view fields in hippocampal CA1 region. (C) Typical photomicrographs of the hippocampal CA1 region from rats that received E2 treatment immediately post-ovariectomy for 10 weeks. TUNEL-positive cells stained red and all other cells were stained blue with DAPI. Scale bar=100 $\mu \mathrm{m}$. (D) TUNEL-positive cells were quantified in each group, $\mathrm{n}=8$ rats per group. Data is presented as the average number of TUNEL-positive cells in five view fields in hippocampal CA1 region. For parts $B$ and $D,{ }^{* * *} \mathrm{P}<0.001$ vs. sham group; ${ }^{*} \mathrm{P}<0.05$ and ${ }^{\# \# "} \mathrm{P}<0.001$ vs. Pla group. Nissl's staining of the whole hippocampus region was conducted in hippocampal sections from sham rats or rats treated with E2, Pla, E2 + G15 or G15 at (E) 10 weeks post-ovariectomy or (F) immediately after ovariectomy for 10 weeks. Rats were treated with G15 at the end of the E2 treatment in the E2 + G15 group. Scale bar=500 $\mu \mathrm{m}$ (upper) and $50 \mu \mathrm{m}$ (lower). (G) E2 replacement at 10 weeks post-ovariectomy did not prevent neuronal death and exert neuroprotection, $n=6-8$ rats per group. (H) Cell counting demonstrated that E2 replacement immediately post-ovariectomy, for 10 weeks, may prevent ischemic damage compared with Pla-treated rats. However, the neuroprotection of E2 was attenuated when G15 was administered at the end of the E2 treatment for 10 weeks, $\mathrm{n}=6-8$ rats per group. For parts $\mathrm{G}$ and $\mathrm{H}$, ${ }^{* * *} \mathrm{P}<0.001$ vs. sham group;

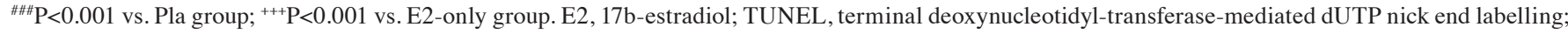
Pla, placebo; G15, G-protein-coupled receptor 30-selective antagonist. 
A

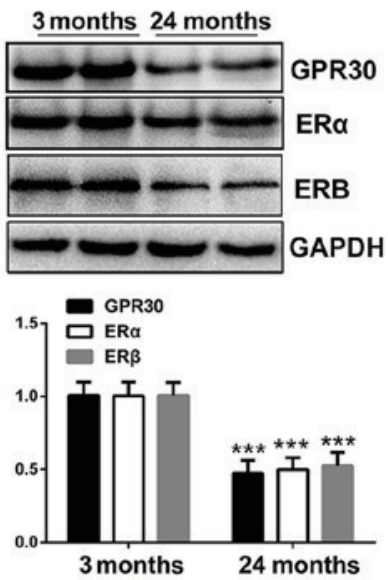

B

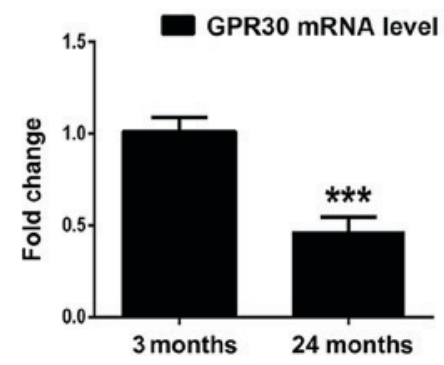

C
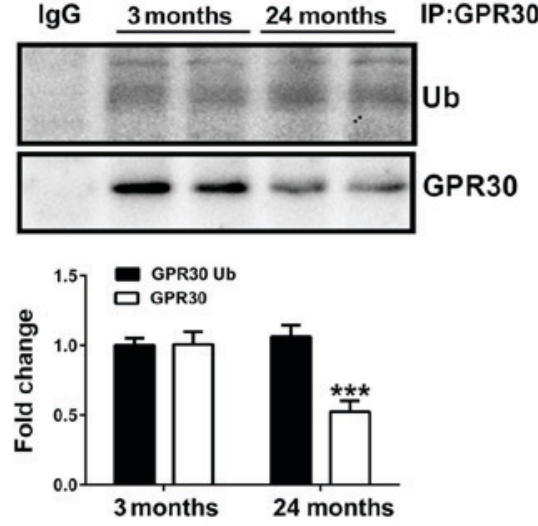

E

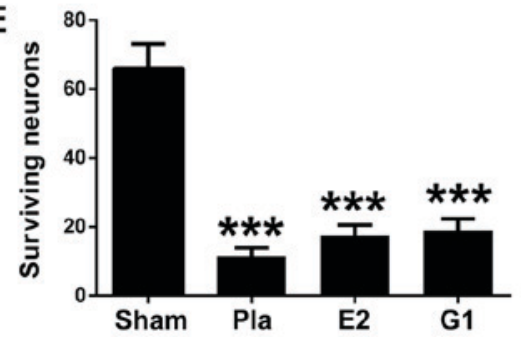

Figure 6. Decreases in GPR30 protein and mRNA expression levels in aging female rats. (A) Western blotting revealed decreased expression levels of GPR30, $\mathrm{ER} \alpha$ and ER $\beta$ protein in the hippocampal CA1 regions of 24-month-old rats compared with 3-month-old rats, $\mathrm{n}=7-8$ rats per group. (B) mRNA expression levels of GPR30 were reduced in 24-month-old rats compared with 3-month-old female rats, n=7-8 per group. (C) Hippocampal CA1 protein samples from the 3- and 24-month-old female rats were immunoprecipitated with anti-GPR30 antibody or nonspecific IgG and subsequently blotted with anti-ubiquitin antibody, $\mathrm{n}=8$ rats per group. For parts $\mathrm{A}-\mathrm{C}$, ${ }^{* * *} \mathrm{P}<0.001$ vs. 3 -month-old rats. (D) Nissl's staining of neurons in the hippocampus of sham rats and Pla-, E2- and G1-treated 24-month-old female rats. Scale bar=500 $\mu \mathrm{m}$ (upper) and $50 \mu \mathrm{m}$ (lower). (E) Quantification of neurons following the various treatments indicated the number of surviving neurons on each $250 \mu \mathrm{m}$ length layer, $\mathrm{n}=7-8$ rats per group. For part E, ${ }^{* * *} \mathrm{P}<0.001$ vs. sham group. GPR30, G-protein-coupled receptor 30; ER, estrogen receptor; IgG, immunoglobulin G; Pla, placebo; E2, 17b-estradiol; G1, GRP30-selective agonist; RT-qPCR, reverse transcription-quantitative polymerase chain reaction; IP, immunoprecipitation; Ub, ubiquitin.

exists for beneficial hormone therapy following menopause, and healthy cell bias which suggested that E2 only yields neurological benefit if it was applied to healthy neurons. These hypotheses suggest that E2 must be administered during perimenopause to observe its neurological benefit within the CA1 region of the hippocampus, which is critical for memory and learning (29). E2 was reported to function in the maintenance of optimal mitochondrial bioenergetics within neurons by promoting their use of glucose as the major energy source via oxidative phosphorylation (30). However, during LTED, E2 was not able to perform this function, which caused neurons to alter their preferred energy source from glucose to ketones (30). In addition, the functions of E2 in enhancing attentional processes, long-term potentiation, choline acetyltransferase activity and rate-limiting enzyme for neurotransmitter acetylcholine synthesis activity were lost during LTED (31). A previous study further demonstrated a marked decrease in ER $\beta$ and ER $\alpha$ following LTED; however, the loss of E2 neuroprotection was associated with reduced ER $\alpha$, but not ER $\beta$, levels within the hippocampal CA1 region (23). The aforementioned findings may not completely explain the loss of E2 neuroprotection within postmenopausal women.

GPR30 was indicated to participate in mediating E2 rapid signaling and neuroprotective effects using in vivo and in vitro models of GCI and middle cerebral artery occlusion or slice cultures $(15,16,18,19,32,33)$. It has previously been reported that the GPR30-selective agonist G1 significantly attenuates glutamate- or oxidative stress-induced neuronal death $(15,34)$. G1 couples to pertussis toxin-sensitive G-proteins and activates multiple signaling pathways, including Src proto-oncogene, mitogen-activated protein kinases and ERKs. Transcriptional regulation via the activation of GPR30 has also been reported (35). However, alterations in GPR30 expression and its role in ischemic stroke during the critical period remain unknown. Furthermore, the difference in GPR30 protein expression levels between the STED and LTED rats remains unclear. The results of the present study indicated that E2 and G1 exerted a neuroprotective effect on STED rats, as reported previously $(18,28)$. However, robust neuroprotection exhibited by $\mathrm{G} 1$ or $\mathrm{E} 2$ within the CA1 region of LTED rats was not detected in the present study. The alterations in GPR30 levels between the STED and LTED rats were further investigated in the present study; a significant decrease was reported in the LTED rats compared with STED rats. The findings of the present study indicated that the loss of E2-associated neuroprotective effects in the CA1 region of the hippocampus may be associated with a decrease in GPR30 expression levels.

To investigate the GPR30 decrease in the CA1 region of the hippocampus of LTED rats, mRNA and ubiquitination levels of GRP30 were analyzed. The RT-qPCR results revealed that the GPR30 mRNA expression levels were significantly decreased within the CA1 region of LTED rats compared with STED rats, while no significant difference 
of the GPR30 ubiquitination was observed between LTED and STED rats, indicating that decreases in GPR30 expression levels may be primarily attributable to the decline in the mRNA expression levels of GPR30. Additionally, E2 treatment initiated 10 weeks post-ovariectomy exhibited no effects in preventing decreases in GPR30 expression levels; E2 was unable to demonstrate robust neuroprotective effects following GCI. However, E2 treatment for 10 weeks during the entire ovariectomy period was able to prevent reductions in GPR30 expression levels and exhibit robust neuroprotective effects. G15, the specific inhibitor of GPR30, attenuated the neuroprotective effects of $\mathrm{E} 2$ within the CA1 region of the hippocampus when administered near the end of E2 treatment for 10 weeks. These results indicated that GPR30 may be an important factor in E2 neuroprotection loss. Collectively, E2 loss following ovariectomy may have contributed to reductions in the mRNA expression levels of GPR30 and E2 sensitivity loss in the CA1 region of the hippocampus. The results of the present study also supported the hypothesis of the critical period as E2 treatment for the entire ovariectomy period was fully neuroprotective.

As ovariectomy in young adult rats removed numerous steroids, including estrogen, progestogen and other factors, LTED did not recapitulate the alterations that occurred in naturally aging female rats. Similar experiments were performed in 3- and 24-month-old female rats in the present study. The 24-month-old female rats were reported to exhibit a decrease in GPR30 levels in the CA1 region of the hippocampus, compared with 3-month-old rats. Furthermore, $\mathrm{ER} \alpha$ and ER $\beta$ also decreased significantly in the 24-month rats compared with 3 month-old rats, which was consistent with a previous report (20). The mRNA expression levels of GPR30 in 24-month-old female rats in the CA1 region of the hippocampus were reduced by $\sim 50 \%$ compared with 3-month-old rats; however, the ubiquitination of GPR30 was not significantly different between the two groups. Further experiments in the present study indicated that neither E2 nor G1 exerted neuroprotective effects in 24-month-old female rats, which may be due to decreases in the protein and mRNA levels of GPR30 in the CA1 region of the hippocampus. These findings indicated that when E2 or G1 was administered in perimenopausal women, the decrease in GPR30 expression levels may be reduced, contributing to robust neuroprotection by E2 treatment.

In conclusion, the present study demonstrated that the decline in GPR30 mRNA expression levels may be responsible for the decrease in GPR30 expression within the hippocampal CA1 region following aging and LTED, which was closely associated with the loss of E2-mediated neuroprotection. In addition, these findings may provide important insights into the underlying mechanism of the critical period hypothesis of E2-associated neuroprotective effects. Although the transcriptional regulation needed further investigation, our present findings suggested GPR30 might exert as a potential novel therapeutic target against ischemic stroke for perimenopausal patients in clinical settings.

\section{Acknowledgements}

Not applicable.

\section{Funding}

The present study was supported by the National Natural Science Foundation of China (grant no. 81200902) and the Natural Science Foundation for Young Scientists of Shaanxi Province (grant no. S2015YFJQ1250).

\section{Availability of data and materials}

The analyzed data sets generated during the study are available from the corresponding author on reasonable request.

\section{Authors' contributions}

GG and TZ designed the study and wrote the protocol. YW, DF and JL completed the experiments, YW established the animal models. SH performed the western blotting and qPCR. YW and DF wrote the first draft of the manuscript. All authors contributed to and approved the final manuscript.

\section{Ethics approval and consent to participate}

The study protocol was approved by the Animal Care and Use Committee of the Fourth Military Medical University.

\section{Consent for publication}

Not applicable.

\section{Competing interests}

The authors declare that they have no competing interests.

\section{References}

1. Brann DW, Dhandapani K, Wakade C, Mahesh VB and Khan MM: Neurotrophic and neuroprotective actions of estrogen: Basic mechanisms and clinical implications. Steroids 72: 381-405, 2007.

2. Rocca WA, GrossardtBR and ShusterLT: Oophorectomy, menopause, estrogen treatment, and cognitive aging: Clinical evidence for a window of opportunity. Brain Res 1379: 188-198, 2011.

3. Shuster LT, Rhodes DJ, Gostout BS, Grossardt BR and Rocca WA: Premature menopause or early menopause: Long-term health consequences. Maturitas 65: 161-166, 2010.

4. Wassertheil-Smoller S, Hendrix SL, Limacher M, Heiss G, Kooperberg C, Baird A, Kotchen T, Curb JD, Black H, Rossouw JE, et al: Effect of estrogen plus progestin on stroke in postmenopausal women: The Women's Health Initiative: A randomized trial. JAMA 289: 2673-2684, 2003.

5. Espeland MA, Rapp SR, Shumaker SA, Brunner R, Manson JE, Sherwin BB, Hsia J, Margolis KL, Hogan PE, Wallace R, et al: Conjugated equine estrogens and global cognitive function in postmenopausal women: Women's Health Initiative Memory Study. JAMA 291: 2959-2968, 2004.

6. Santen RJ, Allred DC, Ardoin SP, Archer DF, Boyd N, Braunstein GD, Burger HG, Colditz GA, Davis SR, Gambacciani M, et al: Postmenopausal hormone therapy: An Endocrine Society scientific statement. J Clin Endocrinol Metab 95 (7 Suppl 1): S1-S66, 2010.

7. Sherwin BB: The critical period hypothesis: Can it explain discrepancies in the oestrogen-cognition literature? J Neuroendocrinol 19: 77-81, 2007.

8. Sherwin BB: Estrogen therapy: Is time of initiation critical for neuroprotection? Nat Rev Endocrinol 5: 620-627, 2009.

9. Craig MC and Murphy DG: Estrogen therapy and Alzheimer's dementia. Ann N Y Acad Sci 1205: 245-253, 2010.

10. Gibbs RB: Estrogen therapy and cognition: A review of the cholinergic hypothesis. Endocr Rev 31: 224-253, 2010. 
11. Moura PJ and Petersen SL: Estradiol acts through nuclearand membrane-initiated mechanisms to maintain a balance between GABAergic and glutamatergic signaling in the brain: Implications for hormone replacement therapy. Rev Neurosci 21: 363-380, 2010.

12. Boulware MI, Kent BA and Frick KM: The impact of age-related ovarian hormone loss on cognitive and neural function. Curr Top Behav Neurosci 10: 165-184, 2012.

13. Daniel JM and Bohacek J: The critical period hypothesis of estrogen effects on cognition: Insights from basic research Biochim Biophys Acta 1800: 1068-1076, 2010.

14. Prossnitz ER and Barton M: The G-protein-coupled estrogen receptor GPER in health and disease. Nat Rev Endocrinol 7: 715-726, 2011.

15. Gingerich S, Kim GL, Chalmers JA, Koletar MM, Wang X, Wang Y and Belsham DD: Estrogen receptor alpha and G-protein coupled receptor 30 mediate the neuroprotective effects of $17 \beta$-estradiol in novel murine hippocampal cell models. Neuroscience 170: 54-66, 2010.

16. Liu SB, Han J, Zhang N, Tian Z, Li XB and Zhao MG: Neuroprotective effects of oestrogen against oxidative toxicity through activation of G-protein-coupled receptor 30 receptor Clin Exp Pharmacol Physiol 38: 577-585, 2011.

17. Zhang B, Subramanian S, Dziennis S, Jia J, Uchida M Akiyoshi K, Migliati E, Lewis AD, Vandenbark AA, Offner H and Hurn PD: Estradiol and G1 reduce infarct size and improve immunosuppression after experimental stroke. J Immunol 184: 4087-4094, 2010.

18. Kosaka Y, Quillinan N, Bond C, Traystman R, Hurn P and Herson P: GPER1/GPR30 activation improves neuronal survival following global cerebral ischemia induced by cardiac arrest in mice. Transl Stroke Res 3: 500-507, 2012.

19. Tang H, Zhang Q, Yang L, Dong Y, Khan M, Yang F, Brann DW and Wang R: GPR30 mediates estrogen rapid signaling and neuroprotection. Mol Cell Endocrinol 387: 52-58, 2014.

20. Zhang QG, Han D, Wang RM, Dong Y, Yang F, Vadlamudi RK and Brann DW: C terminus of Hsc70-interacting protein (CHIP)-mediated degradation of hippocampal estrogen receptor-alpha and the critical period hypothesis of estrogen neuroprotection. Proc Natl Acad Sci USA 108: E617-E624, 2011

21. Stephenson W: Deficiencies in the National Institute of Health's guidelines for the care and protection of laboratory animals. J Med Philos 18: 375-388, 1993.

22. Zhang QG, Wang R, Khan M, Mahesh V and Brann DW: Role of Dickkopf-1, an antagonist of the Wnt/beta-catenin signaling pathway, in estrogen-induced neuroprotection and attenuation of tau phosphorylation. J Neurosci 28: 8430-8441, 2008.

23. Zhang QG, Raz L, Wang R, Han D, De Sevilla L, Yang F, Vadlamudi RK and Brann DW: Estrogen attenuates ischemic oxidative damage via an estrogen receptor alpha-mediated inhibition of NADPH oxidase activation. J Neurosci 29 $13823-13836,2009$
24. Wang R, Tu J, Zhang Q, Zhang X, Zhu Y, Ma W, Cheng C, Brann DW and Yang F: Genistein attenuates ischemic oxidative damage and behavioral deficits via eNOS/Nrf2/HO-1 signaling. Hippocampus 23: 634-647, 2013

25. Shen Y, He P, Fan YY, Zhang JX, Yan HJ, Hu WW, Ohtsu H and Chen Z: Carnosine protects against permanent cerebral ischemia in histidine decarboxylase knockout mice by reducing glutamate excitotoxicity. Free Radic Biol Med 48: 727-735, 2010.

26. Wang R, Zhang X, Zhang J, Fan Y, Shen Y, Hu W and Chen Z: Oxygen-glucose deprivation induced glial scar-like change in astrocytes. PLoS One 7: e37574, 2012.

27. Livak KJ and Schmittgen TD: Analysis of relative gene expression data using real-time quantitative PCR and the 2(-Delta Delta C(T)) method. Methods 25: 402-408, 2001

28. Lebesgue D, Chevaleyre V, Zukin RS and Etgen AM: Estradiol rescues neurons from global ischemia-induced cell death: Multiple cellular pathways of neuroprotection. Steroids 74 : 555-561, 2009.

29. Scott E, Zhang QG, Wang R, Vadlamudi R and Brann D: Estrogen neuroprotection and the critical period hypothesis. Front Neuroendocrinol 33: 85-104, 2012.

30. Yao J, Hamilton RT, Cadenas E and Brinton RD: Decline in mitochondrial bioenergetics and shift to ketogenic profile in brain during reproductive senescence. Biochim. Biophys. Acta 1800: 1121-1126, 2010

31. Bohacek J, Bearl AM and Daniel JM: Long-term ovarian hormone deprivation alters the ability of subsequent oestradiol replacement to regulate choline acetyltransferase protein levels in the hippocampus and prefrontal cortex of middle-aged rats. J Neuroendocrinol 20: 1023-1027, 2008.

32. Gibbs RB, Nelson D and Hammond R: Role of GPR30 in mediating estradiol effects on acetylcholine release in the hippocampus. Horm Behav 66: 339-345, 2014.

33. Liu SB, Zhang N, Guo YY, Zhao R, Shi TY, Feng SF, Wang SQ, Yang Q, Li XQ, Wu YM, et al: G-protein-coupled receptor 30 mediates rapid neuroprotective effects of estrogen via depression of NR2B-containing NMDA receptors. J Neurosci 32: 4887-4900, 2012.

34. Bologa CG, Revankar CM, Young SM, Edwards BS, Arterburn JB, Kiselyov AS, Parker MA, Tkachenko SE, Savchuck NP, Sklar LA, et al: Virtual and biomolecular screening converge on a selective agonist for GPR30. Nat Chem Biol 2: 207-212, 2006.

35. Notas G, Kampa M, Pelekanou V and Castanas E: Interplay of estrogen receptors and GPR30 for the regulation of early membrane initiated transcriptional effects: A pharmacological approach. Steroids 77: 943-950, 2012.

This work is licensed under a Creative Commons Attribution-NonCommercial-NoDerivatives 4.0 International (CC BY-NC-ND 4.0) License. 